Recepción: 20 / 12 / 2018

Aceptación: 28 / 01 / 2019

Publicación: 20 / 03 / 2019

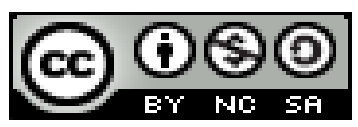

Ciencias de la educación

Artículo de revisión

\title{
Incidencia de la educación inicial, básica y el bachillerato en las instituciones educativas
}

\author{
Incidence of initial, basic education and baccalaureate in educational \\ institutions
}

Incidência de formação inicial, básica e bacharelado em instituições de ensino

Lady D. Vera-Solórzano ${ }^{\text {I }}$ ladyverasolorzano@gmail.com

Luz M. Samaniego-Bautista II mar1978@hotmail.com

Edison C. Maldonado-Alvarado III edisonmaldonado2008@hotmail.com

Andrea C. Pabón-Soria IV andreacarolinapabon@outlook.com

Andrea C. Loachamin-Guachamin ${ }^{\mathrm{V}}$ andre_a00@hotmail.es

Katty A. Chariguaman-Chanaluisa VI kattych2013@live.com

Correspondencia: ladyverasolorzano@gmail.com

I. Máster Universitario en Dirección y Administración de Empresas; Directora de Desarrollo Profesional Educativo. Ministerio de Educación. Zona 2, Ecuador.

II. Máster en Proyectos Socio-Productivos; Directora de Apoyo, Seguimiento y Regulación. Ministerio de Educación, Ecuador. Máster en Educación con mención en Planificación y Proyectos; Director de Planificación. Ministerio de Educación. Zona 2, Ecuador.

IV. Ingeniera Comercial; Analista Zonal de Desarrollo Profesional 2. Ministerio de Educación. Zona 2, Ecuador.

v. Ingeniera Geógrafa en Planificación Territorial; Analista de Planificación. Ministerio de Educación. Zona 2, Ecuador.

VI. Ingeniera Comercial; Analista de Apoyo Seguimiento y Regulación. Ministerio de Educación. Zona 2, Ecuador. 


\section{Resumen}

Esta investigación se centra en caracterizar la incidencia de la educación inicial, básica y el bachillerato en las instituciones educativas, tomando en consideración que el éxito académico depende de los hábitos y técnicas de estudio que se emplean tanto en la institución educativa como en casa. En tal sentido, se evaluó el ambiente y los materiales necesarios para un estudio adecuado y exitoso, sumado a la labor que realizan los docentes mediante su desempeño de formación dentro del sistema educativo y formal, considerando la importancia de la formación inicial y la capacitación continua y permanente de los educadores, de acuerdo a la incidencia en la calidad del trabajo, lo que produce un entorno competitivo que generan atributos para el desempeño del docente en situaciones diversas dentro del campo educativo. El uso de material didáctico permite desarrollar caracteres importantes que intervienen y facilitan el proceso de enseñanza-aprendizaje. Durante el desarrollo de la investigación se observa, que el empleo de materiales didácticos tanto físicos como virtuales, logra despertar el interés de los estudiantes, adecuándose a las características físicas y psíquicas de los mismos, además que facilita la actividad docente al servir de guía. El acceso a los diferentes recursos TIC, ofrecen un entorno mucho más favorable para el aprendizaje y una experiencia docente más dinámica, ofreciendo a los estudiantes herramientas motivadoras que brindan aprendizajes más activos. La incidencia de las redes sociales en la educación de adolescentes se ha transformado en una plataforma de comunicación global, sin embargo, sucede que también en ocasiones se han convertido en distractores para los estudiantes lejos de la misión de educar.

Palabras claves: Hábitos y Técnicas de Estudio; Capacitación Continua; Enseñanza-Aprendizaje; Recursos Tic; Incidencia de las Redes Sociales.

\section{Abstract}

This research focuses on characterizing the incidence of initial, basic and baccalaureate education in educational institutions, taking into account that academic success depends on the study habits and techniques used both in the educational institution and at home. In this regard, the environment and the materials necessary for an adequate and successful study were evaluated, in addition to the work carried out by the teachers through their training performance within the formal and educational system, considering the importance of initial training and continuous training and permanent of the educators, according to the incidence in the quality of the work, which produces a competitive environment that generates attributes for the performance of the teacher in diverse situations within 
the educational field. The use of didactic material allows to develop important characters that intervene and facilitate the teaching-learning process. During the development of the research it is observed that the use of didactic materials, both physical and virtual, manages to awaken the interest of the students, adapting to the physical and mental characteristics of the same, as well as facilitating the teaching activity to serve as a guide. Access to different ICT resources offers a much more favorable environment for learning and a more dynamic teaching experience, offering students motivating tools that provide more active learning. The incidence of social networks in the education of adolescents has become a platform for global communication, however, it happens that they have also sometimes become distractions for students away from the mission of educating.

Keys words: Study Habits and Techniques; Continuous Training; Teaching-Learning; Ict Resources; Incidence of Social Networks.

\section{Resumo.}

Esta pesquisa enfoca a caracterização da incidência da educação inicial, básica e de bacharelado em instituições de ensino, levando em conta que o sucesso acadêmico depende dos hábitos e técnicas de estudo utilizados tanto na instituição de ensino quanto no domicílio. Nesse sentido, foram avaliados o meio ambiente e os materiais necessários para um estudo adequado e bem-sucedido, além do trabalho realizado pelos professores por meio do desempenho de treinamento no sistema formal e educacional, considerando a importância da formação inicial e da formação contínua e permanente dos educadores, segundo a incidência na qualidade do trabalho, o que produz um ambiente competitivo que gera atributos para a atuação do professor em diversas situações dentro do campo educacional. O uso de material didático permite desenvolver personagens importantes que intervêm e facilitam o processo de ensino-aprendizagem. Durante o desenvolvimento da pesquisa observa-se que a utilização de materiais didáticos, tanto físicos como virtuais, consegue despertar o interesse dos alunos, adaptando-se às características físicas e mentais dos mesmos, bem como facilitando a atividade docente para servir. é um guia. O acesso a diferentes recursos de TIC oferece um ambiente muito mais favorável para a aprendizagem e uma experiência de ensino mais dinâmica, oferecendo aos alunos ferramentas motivadoras que proporcionam uma aprendizagem mais ativa. A incidência de redes sociais na educação de adolescentes tornou-se uma plataforma de comunicação global, no entanto, acontece que eles também se tornaram, às vezes, distrações para os estudantes longe da missão de educar. 
Lady D. Vera-Solórzano; Luz M. Samaniego-Bautista; Edison C. Maldonado-Alvarado; Andrea C. Pabón-Soria; Andrea C. Loachamin-Guachamin; Katty A. Chariguaman-Chanaluisa

Palavras chaves: Hábitos e Técnicas de Estudo; Treinamento Contínuo; Ensino-Aprendizagem; Recursos Ict; Incidência de Redes Sociais.

\section{Introducción.}

Las instituciones educativas respecto a su desempeño deben apuntar al éxito académico, pues alrededor de ella deben girar los buenos hábitos de estudio, dentro de ese marco es importante contar con adecuados ambientes generando un clima institucional, contar con los requerimientos para un buen desempeño docente, así como los materiales didácticos y tecnológicos necesarios con el propósito de que el estudio se transforme en una actividad exitosa donde el alumno será el mayor beneficiario.

La educación ha sido un tema de estudio de la academia en forma permanente. Estos abordajes cobran importancia en el marco de los esfuerzos que han hecho distintas organizaciones internacionales como la Organización de las Naciones Unidas [ONU], la Organización para la Educación, la Ciencia y la Cultura [UNESCO], la Organización de Estados Americanos [OEA] y el Fondo de las Naciones Unidas para la Infancia [UNICEF], en pro de mejoras sociales y en busca de condiciones cada vez más dignas para los habitantes de distintos territorios. Esto ha enarbolado entre otras, la bandera de la Educación para Todos [EpT], como uno de los propósitos a mediano plazo para el mejoramiento y la transformación de la educación a nivel mundial y, al mismo tiempo, para que ésta empiece a ser vista no sólo como un escenario de apertura, sino como un contexto participativo, incluyente y construido desde la diversidad. (Bustos G., 2016)

Los sistemas educativos siguen una tendencia generalista con escuelas primarias para las clases bajas en el extremo inferior y escuelas secundarias muy prestigiosas, que preparaban para la universidad, en el extremo superior. El proceso de sistematización impulsó la expansión de 
instituciones intermedias, originalmente de carácter aplicado, que debido a la presión y la aspiración social de padres y profesores tendieron a hacerse más académicas y generalistas, en un intento por aproximarse a las escuelas secundarias de prestigio tradicional. (Viñao, 2002)

Se sabe que la educación en la infancia representa una enorme importancia, esto, debido a que, es en esta etapa de la vida se establecen las bases del desarrollo del individuo y por ende, de la sociedad. Por lo tanto, los principales esfuerzos en la calidad de la formación de los tutores y tutoras, deben estar orientados a quienes trabajan con la infancia. (Guzmán, 2010).

La escuela graduada sirvió para incorporar a la educación a aquellos alumnos de clases sociales desfavorecidas, basándose su organización en distintas ramas y especialidades destinadas a los alumnos de distintas clases y origen social, las escuelas separadas para personas pertenecientes a grupos culturales y minorías étnicas cumplieron el mismo papel en relación a las diferencias por motivos culturales; también la incorporación de la mujer a la escuela pública se produjo separando a las personas de distinto sexo en centros diferentes y, por último, los alumnos categorizados como deficientes fueron escolarizados en la red de centros de Educación Especial. (Parrilla, 2002).

Para la primera década del 2000, algunos artículos evidencian que los diagnósticos cobran mayor cercanía a las necesidades educativas especiales asociadas a trastornos del desarrollo y cómo éstas vienen a incidir en el mundo social de los estudiantes, durante su tránsito por las instituciones educativas. (Montolio, 2008).

En el año 2002, se llevó a cabo en la Habana, Cuba, La Primera Reunión Intergubernamental del Proyecto Regional de Educación para América Latina y el Caribe (2002-2017), para orientar cambios en las políticas y prácticas educativas con miras a los desafíos del siglo XXI. Dichos desafíos se refieren al desarrollo humano, la equidad social y la integración cultural, sin desconocer 
Lady D. Vera-Solórzano; Luz M. Samaniego-Bautista; Edison C. Maldonado-Alvarado; Andrea C. Pabón-Soria; Andrea C. Loachamin-Guachamin; Katty A. Chariguaman-Chanaluisa

que ya en la región se habían adelantado avances en la cobertura, la calidad y la equidad de la educación sin que los resultados fueran los esperados. (UNESCO, 2002)

La incorporación de las TIC en educación tiene como función ser un medio de comunicación, canal de comunicación e intercambio de conocimiento y experiencias, instrumentos para procesar la información, fuente de recursos, instrumento para la gestión administrativa, medio lúdico y desarrollo cognitivo. Todo esto conlleva a una nueva forma de elaborar una unidad didáctica y por ende de evaluar, debido a que las formas de enseñanza y aprendizaje cambian, el profesor ya no es el gestor del conocimiento, sino que un guía que permite orientar al alumno frente su aprendizaje, en este aspecto, el alumno es el "protagonista de la clase", debido a que es él quien debe ser autónomo y trabajar en colaboración con sus pares. (UNESCO, 2005).

La UNESCO, hace referencia a la educación inclusiva como una enseñanza que tenga como propósito desarrollar plenamente el potencial humano, el sentido de la dignidad, la autoestima y el respeto. Los logros de los objetivos del Desarrollo del Milenio y la EpT, en la Conferencia Internacional de Educación, que lleva como título: Educación inclusiva. El camino hacia el futuro. "una educación de calidad debe velar por la participación plena de todos los educandos, con independencia de su sexo, condición económica o social, origen étnico o racial, situación geográfica, necesidades especiales de aprendizaje, edad o religión”. (UNESCO, 2008).

La mayor parte de personas han transitado por las aulas de clase, unos con mayor intensidad que otros, éstas experiencias les han permitido plantear la siguiente interrogante ¿La educación y la formación, son parte del ser humano?, en realidad sí, porque todos los días aprendemos algo; con nuestros amigos, en una institución educativa o en la sociedad, porque "educar es transmitir 
conocimientos, lo que implica un bagaje de información científica y una sólida formación humanista de los niños y jóvenes”. (Flores, 2011).

Como un proceso de abordaje y respuesta a la diversidad de las necesidades de todos los alumnos mediante la participación activa en el aprendizaje y las actividades implícitas de una cultura en pos de reducir la exclusión en el sistema educativo, "implica cambios y modificaciones de contenidos, enfoques, estructuras y estrategias basados en una visión común que abarca a todos los niños en edad escolar y la convicción de que es responsabilidad del sistema educativo regular educar a todos los niños y niñas", (UNESCO, 2005).

La inclusión ha de ser vista como una búsqueda constante de mejores maneras de responder a la diversidad del alumnado,... (ha de buscar) la presencia, la participación y el éxito de todos los estudiantes,... (por tanto) precisa la identificación y la eliminación de barreras... (además ha de enfatizar) en aquellos grupos de alumnos que podrían estar en riesgo de marginalización, exclusión, o fracaso escolar. (Echeita, 2011).

Alcanzar la escolarización básica a los niños en edad escolar y ofrecerles una educación general mínima de 8 a 10 años de duración; superar el analfabetismo, desarrollar y ampliar los servicios educativos para jóvenes y adultos con escolaridad incipiente o sin escolaridad; mejorar la calidad y la eficiencia de los sistemas educativos, y de la enseñanza en general, a través de la realización de las reformas necesarias y del diseño de sistemas efectivos de medición de los aprendizajes (UNESCO, 2011).

Desde hace varios años, los estudiosos del tema educativo vienen insistiendo en la necesidad de fortalecer la formación de los docentes, porque se considera que el maestro es el eje del sistema y debe ser el protagonista de todos los cambios que necesita la educación para constituirse en factor de 
Lady D. Vera-Solórzano; Luz M. Samaniego-Bautista; Edison C. Maldonado-Alvarado; Andrea C. Pabón-Soria; Andrea C. Loachamin-Guachamin; Katty A. Chariguaman-Chanaluisa

desarrollo y de superación. Lamentablemente, a pesar de todas las buenas intenciones, los procesos de formación de los docentes no han sido atendidos por las autoridades educativas; las instituciones formadoras se han debatido entre el desorden, la insuficiencia de políticas educativas y la falta de recursos humanos y financieros. En los últimos años, recién a partir de 2006, se han producido varios cambios que, se espera, contribuyan al mejoramiento del sistema (Fabara, 2013).

Hoy, se evidencia que las calificaciones son sobre 10 puntos y que lo mínimo que se debe tener en los quimestres o semestres es de 7/10, pero esto no significa que en realidad los conocimientos que tienen nuestros estudiantes son aquellos indispensables que necesita el ser humano para defenderse en esta sociedad. El gran problema que presenta la educación ecuatoriana es que no existe una coherencia estructural entre la Educación Inicial, Básica, Bachillerato y la Educación Superior. Barrera, et al., (2017).

\section{Metodología.}

Con el presente trabajo científico se efectuó una revisión de literatura en diferentes bases de datos. Para la realización de la actual investigación, se considero, información confiable sobre trabajos versados en este tema, en los que se estudia la incidencia de la educación inicial, básica y el bachillerato en las instituciones educativas, se utilizó información bibliográfica, misma que se la obtuvo mediante consultas electrónicas, de libros, artículos y revistas científicas.

\section{Desarrollo.}

\section{Hábitos de estudio}

Un hábito es cualquier acto adquirido por la experiencia y realizado regular y automáticamente. Es una práctica o costumbre que realizas con frecuencia. Los antiguos filósofos decían que el hábito es 
una "segunda naturaleza"; eso significa que la naturaleza del hombre se enriquece o empobrece, se perfecciona o se denigra, con el hábito. (Góngora, 2010).

En la Educación Inicial, según los especialistas, "los primeros años corresponden a una edad de oro, en el desarrollo y aprendizaje, por eso deben aprovecharse”. Espinoza, et al., (2013).

Mucho de los problemas referentes al éxito académico en las instituciones educativas, giran alrededor de los hábitos y técnicas de estudio que se emplean en la escuela y en su casa. En este sentido, es muy "importante contar con un adecuado ambiente y materiales necesarios para que el estudio sea una actividad exitosa”. (Brunet F. , 2007).

Los hábitos de estudio se adquieren voluntaria o involuntariamente, originándose en los cuidados de los padres y los maestros, por lo que es fundamental que estos proporcionen, por una parte, medios para que se adquieran los hábitos considerados como buenos o útiles, y por otra, eviten que surjan los incorrectos o perjudiciales para el individuo y la sociedad. (Belaunde, 1994).

Hoy existe una necesidad imperiosa de conocimientos en nuestros estudiantes, pero si no la accionamos de manera lúdica, se pierde la posibilidad de sembrar la semillita del saber en estos niños o niñas, con ello estaría dando paso a que esos aprendizajes sean reforzados y manejados con estrategias metodológicas proactivas en la Educación Básica donde el niño empieza a leer y escribir de manera más profunda. Barrera, et al., (2017).

Las técnicas de estudio son estrategias, procedimientos o métodos, que se ponen en práctica para adquirir aprendizajes, ayudando a facilitar el proceso de memorización y estudio, para mejorar el rendimiento académico (Brunet G. , 2005). El aprendizaje puede relacionarse con el manejo de un contenido teórico o el desarrollo de habilidades para dominar una actividad práctica. Borda \& 
Pinzón, (1995). La lectura puede activar más de 20 procesos intelectuales, además se convierte en la entrada a un mundo lleno de aventuras y conocimientos. (Crespo, 2010)

Es por ello que hay que incorporar las destrezas del escuchar, hablar, leer y escribir, potencializando el desarrollo de destrezas con criterios de desempeño que maneja el Ministerio de Educación, con este enfoque la continuidad en el Bachillerato permitirá desarrollar macro destrezas, en donde el estudiante tenga la posibilidad de aplicar las operaciones intelectuales en cada forma del pensamiento, así: pensamiento reflexivo, analítico, lógico, crítico, sistemático, analógico, creativo, deliberativo y práctico. Barrera, et al., (2017).

La interculturalidad como base de la diversidad, debe ser entendida como el aprendizaje entre niños, jóvenes y adultos pertenecientes a diferentes espacios culturales, es esa interacción que permite el intercambio y el enriquecimiento de pensamientos y visiones respecto a algo con base en actitudes de respeto y comprensión del otro. (Giménez, 2003).

La Educación es sinónimo de aprendizaje por ello cada uno de los niveles educativos que transitamos deben ser pertinentes, coherentes y praxitivos, de tal manera que exista una verdadera conexión de conocimientos y el anclaje de los aprendizajes, para que estos se tornen auténticos aprendizajes significativos. Barrera, et al., (2017).

Es importante destacar que el ambiente e aprendizaje so sólo se refiere al contexto físico y recursos materiales, también implica aspectos psicológicos que son sumamente importantes en el éxito o el fracaso de proyectos educativos. Puede generarse un ambiente propicio para la expresión abierta a la diversidad de opiniones o puede establecerse un ambiente poco tolerante y que imponga puntos de vista; así mismo puede generarse un espacio que motive la participación activa de los estudiantes o que la inhiba. (Herrera, 2010). 


\section{Clima institucional}

El clima organizacional constituye el medio interno de una organización, la atmósfera psicológica característica que existe en cada organización, el clima se percibe en diferentes maneras por diferentes individuos. (Chiavenato, 1984)

Está conformado por elementos estructurados, personales y funcionales de la institución y todos ellos integrados, confieren un estilo especial a la institución que dependerá de los resultados que se produzcan en la institución. En la escuela, en la cual estaría definido a partir de todas aquellas personas que constituyen la comunidad escolar: el director, los profesores, personal administrativo, auxiliar, los alumnos y los padres de familia. El clima de una escuela resulta del tipo de programa, de los procesos utilizados, de las condiciones ambientales que caracteriza la escuela como una institución y como un agrupamiento de alumnos de los departamentos, del personal, de los miembros de la dirección. Cada escuela posee un clima propio y distinto .El clima determina la calidad de vida y la productividad de los profesores y de los alumnos. (Rodríguez, 2004).

En un ambiente positivo de respeto de relaciones afectivas habrá mayores posibilidades de estimular al niño para aprender, otra aspecto son las posibilidades comunicativas que se genera a partir de las tareas diferentes que el docente planifica. Estas formas de comunicación no deben de ser autoritarias ni formales sino afectivas, de cooperación, y a la vez deben ser armoniosas y agradables. "Su ejemplo y el ser portador de las normas que trata de desarrollar, así como el lenguaje coloquial y afectivo que utiliza se constituyen en elementos importantes del clima. (Valdés, 2004).

La escuela, como cualquier otra institución social, desarrolla y reproduce su propia cultura específica. Entiendo por tal cultura el conjunto de significados y comportamientos que genera la escuela como institución social. Las tradiciones, costumbres, rutinas, rituales e inercias que estimula 
Lady D. Vera-Solórzano; Luz M. Samaniego-Bautista; Edison C. Maldonado-Alvarado; Andrea C. Pabón-Soria; Andrea C. Loachamin-Guachamin; Katty A. Chariguaman-Chanaluisa

y se esfuerza en conservar y reproducir la escuela condicionan claramente el tipo de vida que en ella se desarrolla y refuerzan la vigencia de valores, expectativas y creencias ligadas a la vida social de los grupos que constituyen la institución escolar. La escuela no puede entenderse como un mecanismo objetivo de precisión, independientemente del contenido de las tareas y relaciones en las que se implica. La respuesta organizativa de la escuela debe ser ofensiva, es decir, crear un clima de intercambio y experiencia donde tenga cabida la complejidad y la incertidumbre vividas desde la cooperación democrática. (Pérez, 1998).

La habilidad de una organización para utilizar sus recursos en la realización de sus actividades, en la gestión de procesos se examina la manera en que la organización maneja sus relaciones humanas y sus interacciones relacionadas con el trabajo. La estructura identifica los vínculos entre la forma como se rige una organización y su misión, así como las funciones que desempeñan las personas y las finanzas en las actividades cotidianas de la organización, en la cual la capacidad de la organización maneja sus relaciones externas como relaciones interinstitucionales. Lusthaus, et al., (2002).

La dinámica institucional es vista desde una perspectiva psicológica y social, en la cual la institución educativa es un órgano donde las personas actúan y en donde se plantean dificultades en términos de problemas y trabajan para su solución. Ello no se da solo en situaciones críticas o de riesgo, sino también en condiciones óptimas y favorables. El grado de la dinámica, el tipo de movimiento, está dado por la existencia de estrategias y mecanismos a utilizar y la capacidad de acción que adopten en la toma de decisiones que se realiza en la institució. (Nava, 2009).

Ministerio de Educación, Ecuador (2010). Se establecen los escenarios de actuación, que son las instituciones educativas públicas y privadas de educación básica, como: 
- Centros infantiles, jardines infantiles.

- Escuelas públicas y privadas hispanas y bilingües.

- Unidades educativas.

- Direcciones Provinciales de Educación.

- Instituciones de capacitación docente.

- Centros de asesoría pedagógica y ONG.

\section{Desempeño docente}

Es el conjunto de acciones que realiza el maestro, durante el desarrollo de su actividad pedagógica, que se concretan en el proceso de cumplimiento de sus funciones básicas y en sus resultados, para lograr el fin y los objetivos formativos del nivel educativo donde trabaje. Estas acciones tienen, además, un carácter consciente, individual y creador. (Torres, 2008).

El docente debe conocer el contenido de lo que enseña y el modo de cómo ello puede tener sentido para sus alumnos, el docente desde que es un profesional de la docencia se ha preparado y posee conocimientos los cuales pone en práctica al iniciar su clase, el docente conoce el tema a tratar, el investiga, se informa debe de estar de acuerdo con los avances y cambios que la ciencia exige, debe estar a la vanguardia del conocimiento, aunque este término resulte triado, debe siempre utilizar un lenguaje adecuado al nivel que enseña, un lenguaje comprensible y que promueva al diálogo y al aprendizaje de los estudiantes, el docente entrega lo que sabe pensando solo en lo que los alumnos requieren de él. Debe tener conocimientos sobre las disciplinas académicas con las que debe lograr que los alumnos construyan sus aprendizajes, lo que le permita manejar información sobre las características generales del grupo educativo e individualidades de cada uno de los estudiantes. (Hernández, 1999). 
Lady D. Vera-Solórzano; Luz M. Samaniego-Bautista; Edison C. Maldonado-Alvarado; Andrea C. Pabón-Soria; Andrea C. Loachamin-Guachamin; Katty A. Chariguaman-Chanaluisa

El discurso educativo se ha ido modernizando y se modificó a través del tiempo, pero las prácticas docentes no han cambiado. Si bien el docente es la figura clave en la puesta en marcha de cualquier cambio, en la historia de las reformas educativas se lo ha ubicado en un lugar secundario. Elichiry, (2000). El Desempeño docente es el eje que moviliza el proceso de formación dentro del sistema educativo formal. Se hace necesario el análisis y la evaluación del desempeño docente desde la cotidianidad, de un modo concreto y encarnado. (Estrada, 2013)

El tiempo destinado a la profesionalización del cuerpo docente, a propiciar su desarrollo académico, a procurar el comportamiento progresivamente autónomo de las comunidades liceales. A través de ella se pretende que los profesores trabajen en colaboración, estableciendo acuerdos, abordando tareas que atañen al centro en su totalidad, procurando encontrar en conjunto estrategias pedagógicodidácticas para emplear en las aulas, resolver problemas organizacionales del centro, etc. En ella los actores participantes tienen la posibilidad de tomar decisiones, de desarrollar la autonomía, de reflexión y creación, en suma, de lograr mayor responsabilidad de los centros por los aprendizajes de los alumnos y por el de ellos mismos. (Romero, 2002).

Según (Robalino, 2007). La formación inicial y la capacitación continua y permanente de los educadores es uno de los factores que más inciden en la calidad de su trabajo, pese a ello, en la mayoría de países latinoamericanos persisten aún algunos problemas, mismos que son considerados un verdadero desafío para el eficiente desempeño de los docentes:

- Predominio de enfoques y modelos tradicionales en las instituciones formadoras de docentes.

- $\quad$ Déficit en la calidad de los formadores de formadores.

- Desarticulación entre formación inicial y formación permanente. 
- Ausencia de espacios de coordinación entre los responsables de la formación de los docentes.

- Reformas centradas en la capacitación\| sin incidencia en la formación inicial.

- Capacitación, entendida como la sucesión de eventos organizados desde la oferta y no desde la demanda.

- Capacitación a las personas aisladamente y no a los equipos docentes.

- Asistencia a cursos de perfeccionamiento por el puntaje para el ascenso de categorías más allá de la utilidad para una función específica.

- Carencia de apoyo técnico, acompañamiento, monitoreo y evaluación al trabajo docente al interior de las propias instituciones educativas.

- Insatisfacción por los contenidos y la pertinencia de los procesos de formación.

- Débil incorporación de las tecnologías de información y comunicación a la formación de maestros.

- Ausencia de mecanismos institucionalizados de evaluación a la calidad de la formación inicial y en servicio de los docentes.

Para el Ministerio de Educación, Ecuador (2010) los Estándares de Desempeño Docente establecen cuatro grandes dimensiones que contienen las áreas de lo que un docente de calidad debe desarrollar en su desempeño y que influyen en los aprendizajes de los estudiantes. Estas grandes dimensiones son:

1. Desarrollo Curricular.

2. Gestión del Aprendizaje.

3. Desarrollo Profesional.

4. Compromiso Ético. 


\section{El ámbito de las competencias}

El nuevo enfoque de educación determina qué competencias se pretenden formar, en qué contexto, bajo qué fines, en qué espacios, mediante qué tipo de organización curricular, con qué estrategias metodológicas y con qué indicadores e instrumentos de evaluación. La formación basada en competencias establece este ámbito para trascender de la parcelación y la fragmentación a una concepción educativa integradora para hacer frente a la incertidumbre de la sociedad globalizada y en continuo cambio. Las competencias generan atributos para el desempeño en situaciones diversas dentro del campo educativo, tienen una relación dinámica con todos los actores que intervienen en el proceso de formación: instituciones educativas, sector laboral, familiar y sociedad. (Rivadeneira, 2012).

Se comprende que la calidad de los programas de formación no puede lograrse solamente a partir de recetas técnicas o proposiciones de expertos, por el contrario, se requiere del concurso de los distintos actores sociales y políticos como así también de los destinatarios asumiendo una perspectiva integradora y global. El ámbito de las competencias que preferentemente adquiere un profesor en los programas de aprendizaje profesional identifica: primero la colaboración entre profesores para la planificación, Segundo la meta explícita de mejorar el logro de los estudiantes, Tercero la atención al pensamiento de los estudiantes, y Cuarto el acceso a ideas, métodos alternativos y oportunidades de observación de prácticas eficaces. (Villalobos, 2010).

La Dirección Ejecutiva del Consejo Nacional de Educación Superior (CONESUP) emitió el Acuerdo N. ${ }^{\circ} 264$ de 07 de abril de 2005, mediante el cual se establece el Diseño Curricular por Competencias para el Profesor de Educación Básica, en el que se determinan los objetivos de la 
formación, el perfil por competencias, los escenarios de actuación y la Matriz Curricular para alcanzar la formación de este docente. Ministerio de Educación -. (CONESUP, 2006).

Los Objetivos generales de la formación son:

- «Formar un profesional de la docencia con preparación humanística, científica y tecnológica, que garantice un servicio educativo de calidad.»

- «Consolidar en el futuro profesor de Educación Básica un comportamiento ético, centrado en el interés de educar a la niñez y a la juventud, en la investigación y la innovación educativa, comprometido con el desarrollo socioeconómico del país.»

Las competencias principales establecidas en la misma disposición legal son:

- Mediador de aprendizajes significativos y funcionales, potenciando las habilidades del pensamiento en forma reflexiva, crítica y creativa.

- Investigador técnico crítico de la realidad educativa, aplicando procesos cuantitativoscualitativos, con rigor científico y ético.

- Diseñador del currículo en su nivel de desempeño, en función del modelo educativo y pedagógico, las exigencias del entorno, con criterio innovador y participativo.

- Gestor de la institución educativa de calidad, en función de procesos administrativos, con liderazgo y visión de futuro.

- Evaluador de logros en el proceso enseñanza-aprendizaje en el ámbito institucional y de aula en forma criterial y holística.

- Promotor de la participación comunitaria, liderando procesos de integración y consensos, con respeto, solidaridad y equidad. 
- Generador de su desempeño profesional, ético, con valores y principios de convivencia pacífica y práctica de los derechos humanos.

En esta directriz legal se determinan las competencias específicas para cada competencia general y se sistematizan estas por niveles, de manera que al completar el sexto nivel, debe estar en capacidad de:

- Aplicar los conocimientos teóricos en la práctica educativa (ASERO).

- Relacionar la experiencia práctica con las teorías, a fin de buscar soluciones que contribuyan al desarrollo educativo de las comunidades.

- Demostrar una formación profesional y ética en su práctica docente, con capacidad de autoevaluación y rendición de cuentas a la sociedad.

- Aplicar toda su formación en el trabajo de graduación.

Competencias que constituyen el marco curricular común del Sistema Nacional de Bachillerato: “Que entre las competencias a que se refiere el presente Acuerdo se concluyó que las competencias genéricas son las que todos los bachilleres deben estar en capacidad de desempeñar; las que les permiten comprender el mundo e influir en él; les capacitan para continuar aprendiendo de forma autónoma a lo largo de sus vidas, y para desarrollar relaciones armónicas con quienes les rodean, así como participar eficazmente en los ámbitos social, profesional y político. Dada su importancia, dichas competencias se identifican también como competencias clave y constituyen el perfil del egresado del Sistema Nacional de Bachillerato". SEP, (s/f).

Debe enunciarse en forma explícita la competencia en el manejo de información y no ser parte aislada de otras competencias genéricas a fin de que se observen sus indicadores en forma clara y 
puedan ser desarrollados a través de la preparación de programas educativos, tal como sucede en el sistema educativo de otros países. (Gómez, 2011).

Las competencias como enfoque educativo están siendo adoptadas por los docentes como nuevo modelo para el diseño del currículo, la formación y el desarrollo profesional. Por ello se utilizan descriptores como: aprendizaje por competencias y currículo basado en competencias. De acuerdo a este enfoque la competencia se define como conocimientos, habilidad y actitud para satisfacer con éxito, exigencias complejas en un contexto determinado. Para ello el foco de atención son los resultados que se obtienen en el ejercicio. Las competencias, en este sentido son funcionales, eso le ha dado una interpretación de capacidad cognitiva, habilidad cognitiva y modelo de actuación idóneo, representando una capacidad de movilizar varios recursos cognitivos para hacerle frente a situaciones complejas. (Rivadeneira, 2012).

\section{Materiales didácticos y tecnológicos}

Este primer acuerdo sobre la definición de los materiales educativos contribuyó a tener claridad conceptual cuando nos referimos a ellos, a la certeza de saber que nombramos el mismo objeto de estudio. A partir del análisis de distintos textos y autores (Acuña Limón (comp) 1995, Area Moreira 1989, Arévalo y Luviano 2003, Cabero 1990, Davis y Krajcik

2005, Rodríguez y Montero 2002, Sancho Gil, y otros), pudimos constatar que no existen definiciones y taxonomías únicas y que a una misma categoría se les aplican diferentes términos, tal es el caso de "material didáctico" o "recurso didáctico", ello nos llevó a la necesidad de elaborar una clasificación en la que estuviéramos de acuerdo todos y que pudiéramos identificar las mismas categorías. (SEP, 2006). 
Lady D. Vera-Solórzano; Luz M. Samaniego-Bautista; Edison C. Maldonado-Alvarado; Andrea C. Pabón-Soria; Andrea C. Loachamin-Guachamin; Katty A. Chariguaman-Chanaluisa

El autor (Vargas, 2003) expresa que, los materiales didácticos sirven de apoyo para la construcción del conocimiento, por consiguiente es necesario considerar los siguientes aspectos para su uso:

- Reconfiguración del aula: espacio donde se desarrollan las actividades de aprendizaje.

- Rol y perfil del educador: el docente trabaja en forma dinámica e interactiva, facilitando el proceso de enseñanza - aprendizaje.

- Conocimiento de los procesos evolutivos del desarrollo de los niños y niñas: los docentes deben conocer las teorías del desarrollo psicológico y los ritmos de aprendizaje.

- La actividad lúdica: el juego permite la relación del hombre con los demás, con la naturaleza y consigo mismo. Esta ayuda a descubrir reglas, normas, así como situaciones lógicas en forma natural.

- Consideraciones psicopedagógicas para el uso del material educativo: los docentes deben ayudar a desarrollar, a través de las estrategias de aprendizaje, los procesos cognitivos, psicomotores, socio-afectivos.

El uso de material didáctico, en el trabajo de aula, permite desarrollar caracteres importantes en los niños y niñas, como la Expresión creativa; mediante ella pueden relacionar objetos y crear cosas nuevas; y el Pensamiento lógico, que favorece la estructuración mental y conocimiento del entorno. Para desarrollar la ejercitación psicomotora, los docentes utilizan diversos materiales didácticos como el Plantado, Ensartado, Tarjetas, Encaje Plano, ya que estos ayudan a que los niños/as tomen conciencia de su propio cuerpo y de su coordinación psicomotriz. Uno de los aspectos fundamentales que debe desarrollarse en el proceso de enseñanza - aprendizaje es el pensamiento lógico y para ello los maestros de las escuelas utilizan de manera significativa el Rompecabezas, así como la Lotería y el Dominó. Los docentes trabajan con los niños/as desarrollando la expresión artística en el medio educativo con Títeres, Caritas pintadas, Disfraces y Antifaces, los cuales 
permiten vencer la timidez, desarrollar el lenguaje, expresar sentimientos y emociones. Freré, \& Saltos, (2013).

Entiende por material educativo al conjunto de medios materiales que intervienen y facilitan el proceso de enseñanza-aprendizaje. Estos materiales pueden ser tanto físicos como virtuales, asumen, despertar el interés de los estudiantes, adecuarse a las características físicas y psíquicas de los mismos, además que facilitan la actividad docente al servir de guía; asimismo, tienen la gran virtud de adecuarse a cualquier tipo de contenido. (Ogalde, 2008).

Finalmente como expresa (SEP, 2006) optamos por una típica clasificación que hace referencia al soporte físico de los materiales educativos:

1. Materiales Impresos, Libros de texto, manuales, libros para el maestro, ficheros didácticos, etcétera.

2. Materiales Audiovisuales, Videos, diaporamas, películas, programas de televisión, programas de radio, audiocintas, y otros.

3. Materiales Informáticos, Discos compactos, páginas WEB, software educativo, interactivos, y sus derivados.

4. Materiales Objetuales o Concretos, Figuras geométricas, títeres, simuladores, y demás materiales de apoyo.

5. Materiales para la Gestión o Periféricos, Currículum, instrumentos de evaluación, listas de asistencia y calificaciones, proyectos escolares, y similares.

El juego con materiales didácticos tanto estructurados, como no estructurados, ofrece a los niños y a las niñas, la oportunidad de combinar actividad y pensamiento, desarrollar su curiosidad, compartir 
Lady D. Vera-Solórzano; Luz M. Samaniego-Bautista; Edison C. Maldonado-Alvarado; Andrea C. Pabón-Soria; Andrea C. Loachamin-Guachamin; Katty A. Chariguaman-Chanaluisa

experiencias, sentimientos y necesidades, articular la realidad y la fantasía, el conocimiento y la emoción, afianzar su autonomía y autoestima, crear, indagar, observar, y sobre todo relacionar los nuevos descubrimientos con experiencias vividas y así generar nuevos conocimientos. (Rivera, 2011).

Los materiales didácticos son útiles o tienen valor cuando sirven de mediadores para el planeamiento, trabajo y aprendizaje, resolución de problemas, conocimiento de la realidad cotidiana, creación y recreación, iniciativas, participación de los niños/as, investigación y otros. El material didáctico es aquel que reúne medios y recursos que facilitan la enseñanza y el aprendizaje. Suelen utilizarse dentro del ambiente educativo para facilitar la adquisición de conceptos, habilidades, actitudes y destrezas. Por eso, un libro no siempre es un material didáctico. Por ejemplo, leer una novela sin realizar ningún tipo de análisis o trabajo al respecto, no supone que el libro actúe como material didáctico, aun cuando puede aportar datos de la cultura general y ampliar la cultura literaria del lector. (Cárdenas, 2008)

Los Recursos Didácticos son herramientas de suma importancia, que facilitan y apoyan el proceso de enseñanza aprendizaje, cuyo objetivo de su uso es hacer más claros y accesibles los contenidos. Recursos didácticos son un conjunto de elementos que facilitan la realización del proceso enseñanza aprendizaje. Estos contribuyen a que los estudiantes logren el dominio de un contenido determinado. Y por lo tanto, el acceso a la información, la adquisición de habilidades, destrezas y estrategias, como también a la formación de actitudes y valores. (Pilco, 2013).

Son diversas las funciones que los materiales didácticos pueden tener, una de las principales es el reforzar lo que se ha enseñado en clase, de modo que facilite el aprendizaje de los alumnos. Los materiales didácticos, la mayoría, proporcionan información, a través de libros, videos y programas 
informáticos, guiando al estudiante y ayudándolo a crear y aplicar nuevos conocimientos ejercitando habilidades. No obstante, se debe tener en cuenta que los materiales didácticos no solamente sirven para transmitir información sino también funcionan como mediadores entre la realidad y los estudiantes, y mediante sus sistemas simbólicos desarrollan habilidades cognitivas en sus usuarios. Algunas otras funciones de los materiales didácticos son Motivar, Facilitar la adquisición de nuevos conocimientos y Apoyar la evaluación y el Reforzamiento del Aprendizaje. Bautista, et al., (2014).

Con la incorporación de las nuevas tecnologías en todos los aspectos de la vida y la sociedad misma está demandando nuevos modelos de enseñanza y aprendizaje. El modelo de educación centrado en la enseñanza, donde el protagonista es el profesor, deja paso a un sistema basado en el aprendizaje, donde el alumno es el responsable de su propio proceso de aprendizaje y el profesor debe buscar y utilizar la metodología y los medios más adecuados que ayuden al alumno en ese proceso. García \& Lacleta, (2007).

Debe tenerse en cuenta que las posibilidades que nos brindan las nuevas tecnologías como herramienta didáctica, son de igual importancia y es necesario aprovechar todas sus potencialidades para formar seres humanos más justos, más capaces, más cooperativos, los que nos llevaría a afirmar que lo importante no es la tecnología como tal sino que los actores formadores puedan hacer del elemento tecnológico, para humanizarla. (Ossa, 2002).

En relación a los talleres impartidos y el impacto estudiado, se puede concluir que los profesores no asumen como parte de su práctica educativa la función de utilizar la biblioteca, asimismo confunden aún después de haber tomado los talleres, las fuentes de información y su uso. Del mismo modo no redactan en forma correcta la actividad de aprendizaje, en muchos casos existe ambigüedad y en relación a las estrategias son pocos los docentes que las manejan, en otros casos cuando las elaboran 
Lady D. Vera-Solórzano; Luz M. Samaniego-Bautista; Edison C. Maldonado-Alvarado; Andrea C. Pabón-Soria; Andrea C. Loachamin-Guachamin; Katty A. Chariguaman-Chanaluisa

omiten alguno de los puntos revisados, por ejemplo la fuente recomendada para el trabajo y el producto docente solicitado. Se puede concluir que los talleres no cambiaron el trabajo de los docentes con la biblioteca y sus alumnos. (Gómez, 2011).

Crear un ambiente virtual no es trasladar la docencia de un aula física a una virtual, ni cambiar el pizarrón por un medio electrónico, o concentrar el contenido de una asignatura. Se entiende por ambiente virtual de aprendizaje "al espacio físico donde las nuevas tecnologías tales como los sistemas satelitales, el internet, los multimedia, y la televisión interactiva entre otros, se han potencializado rebasando al entorno escolar tradicional que favorece al conocimiento y a la apropiación de contenidos, experiencias y procesos pedagógico-comunicacionales. Están conformados por el espacio, el estudiante, el asesor, los contenidos educativos, la evaluación y los medios de información y comunicación”. (Ávila, 2011).

¿Materiales informáticos o Tecnologías de la información y la comunicación? Hablar de materiales educativos informáticos remite y limita el análisis a productos concretos como pueden ser un disco compacto multimedia, un software o programa educativo o un sitio web. Sin embargo, para los fines de este texto, necesitamos partir de un enfoque más amplio que permita presentar y analizar no sólo los materiales sino también los dispositivos tecnológicos, servicios y contenidos generados como parte de los proyectos de incorporación de las Tecnologías de la Información y la Comunicación (TIC) que se han implementado en las escuelas públicas de educación básica del país. (SEP, 2006).

El acceso a los diferentes recursos TIC, los materiales y programas en el aula pueden ofrecer un entorno mucho más favorable para el aprendizaje y una experiencia docente más dinámica. También la utilización de diversos contenidos digitales de buena calidad enriquece el aprendizaje y puede, a través de simulaciones y animaciones, ilustrar conceptos y principios que de otra manera sería 
difíciles de comprender para los estudiantes. De esta misma manera, las TIC para los estudiantes son fuertemente motivadoras y brindan aprendizajes más activos, su uso en el aprendizaje basado en proyectos y en trabajos grupales permite tener acceso a recursos, lo cual conlleva a una enseñanza más creativa tanta para los docentes como para los estudiantes. León, \& Pacheco, (2014).

El uso de las NTIC, no incluye mención alguna explícita, para usar las bibliotecas impresas o digitales, o comentario alguno de que estas pueden contribuir al desarrollo de competencias en información para favorecer el uso de las NTIC en la educación. Existen grandes riesgos al utilizar, sin criterio documental académico, la información del World Wide Web para generar información para el aprendizaje. La información producida queda en espacios de tecnología educativa, para su uso o reuso sin organización documental, con lo que se pierden oportunidades de recuperación de la información, en conjunto con otros documentos publicados. (Gómez, 2011).

No hay duda de que el uso de material didáctico facilita la enseñanza y constituye un elemento auxiliar en el proceso de aprendizaje funcionando como mediador en la educación de los estudiantes. Es por eso que deben utilizarse materiales didácticos que se presenten mediante las herramientas tecnológicas que son de uso común para los estudiantes, materiales innovadores bien pensados y fabricados especialmente para enseñar y aprender de manera que capten la atención de los estudiantes y les permitan llegar a adquirir determinados conceptos y contribuir así al desarrollo de su pensamiento lógico al mismo tiempo que se sientan motivados con su aprendizaje. Con el uso de los distintos materiales didácticos a través de las TIC's se puede brindar una educación de calidad a un mayor número de estudiante, la institución educativa debe estar preparada para ofrecer esta educación, con un equipo físico, técnico y tecnológico, capaz de afrontar los retos de la nueva era. Bautista, et al., (2014). 
Lady D. Vera-Solórzano; Luz M. Samaniego-Bautista; Edison C. Maldonado-Alvarado; Andrea C. Pabón-Soria; Andrea C. Loachamin-Guachamin; Katty A. Chariguaman-Chanaluisa

Uno de los grandes desafíos de las TIC es el rol que tiene el docente con la implementación de estas nuevas tecnologías, ya que la gran mayoría realizo sus estudios de grado cuando todavía no se habían incorporado, por lo tanto es un tema suma importancia en la mayoría de los planes globales y debería ser un tema de discusión en los lugares donde todavía que no estén instalados. En un documento de la Unesco titulado "Formación docente y las tecnologías de Información y Comunicación. Estudios de casos en Bolivia, Chile, Colombia, Ecuador, México, Panamá, Paraguay y Perú (agosto de 2005)" destaca: que un docente que no maneje las TIC está en desventaja con relación a los alumnos. León, \& Pacheco, (2014).

La tecnología avanza en la vida cotidiana más rápido que en la escuelas, inclusive en zonas alejadas y pobres servicios básicos deficitarios, es por ello que desafortunadamente la sociedad moderna no ha sido capaz de imprimir el mismo ritmo a los cambios que ocurre en la educación. Actualmente, todavía existe un importante número de escuelas que no poseen computadoras, acceso a internet, lo cual esto no necesariamente quiere decir que los estudiantes no estén siendo usuarios de juegos de video, aparatos de audio, telefonía celular e internet. En el campo de las tecnologías los estudiantes de todas maneras las aprenden y utilizan otros contextos. La incorporación de las TIC a la formación docente es un imperativo, tanto para su propia formación como para el aprendizaje de los alumnos, esto no solo implica apoyar a los docentes a que conozcan y manejen los equipos tecnológicos, sino el impacto que influye en su aprendizaje, su uso adecuado, potencialidades y limites. León, \& Pacheco, (2014).

"El concepto de Inclusión Digital se traduce, en contextos escolares, en conseguir la máxima utilización de los recursos informáticos tanto para atender al alumnado con necesidades educativas específicas, como para la normalización de las TIC de uso común (diseño para todos), y la preparación/formación del profesorado en su transformación, uso y aprovechamiento, contemplando 
la adquisición y adaptación de hardware y software adecuado a las necesidades de este alumnado; garantizando la disponibilidad de tecnologías de ayuda a la comunicación aumentativa para los alumnos que lo precisen; fomentando el diseño accesible en la elaboración de recursos (tanto comunes como específicos) multimedia y servicios de red e Internet; e impulsando la formación y la creación de grupos de trabajo, seminarios y proyectos de innovación e investigación educativa cuyas líneas de acción se centren en la utilización y/o el análisis, catalogación y evaluación de las TIC en la atención a la diversidad”. Soto, y Fernández, (2003).

Las TIC deben ser vistas como elementos mediadores y didácticos que favorecen la comunicación, la enseñanza y los aprendizajes. Para la selección de recursos, estrategias de enseñanza y materiales didácticos es importante tener en cuenta todo el contexto, curricular y sociocultural; estos aspectos dependen del docente, pero se requieren cambios de actitud en las formas de enseñar (metodología y estrategias) y de aprender de los estudiantes; por ello, no se deben desconocer las ventajas de las TIC en el aula, deben considerarse un aliado, un "amigo", del proceso formativo para la formulación de Proyectos de TE. Sería pertinente ayudar a identificar y clasificar los Recursos de Información, de Colaboración y de Aprendizaje, para apoyar la tarea del docente y disminuir la confusión al relacionar técnicas con estrategias y recursos con herramientas. (Rivero, 2013).

Futuros estudios podrían evidenciar, con la práctica, la incidencia de la selección de tecnologías, a partir de los criterios y factores propuestos en esta investigación, con estudios experimentales en aulas, áreas o niveles formativos, unido a la selección de recursos de las TIC para la interacción, el aprendizaje colaborativo y el desarrollo de la autonomía del estudiante, como las Webquest, las Wiki o los Blog. La investigación se realizó en un colegio oficial; sería pertinente un estudio comparativo con instituciones del sector privado, para determinar si las diferentes características contextuales, humanas, físicas, técnicas y tecnológicas, inciden en la selección de estrategias, 
Lady D. Vera-Solórzano; Luz M. Samaniego-Bautista; Edison C. Maldonado-Alvarado; Andrea C. Pabón-Soria; Andrea C. Loachamin-Guachamin; Katty A. Chariguaman-Chanaluisa

recursos y materiales didácticos, o si se depende de otras situaciones o variables no contempladas en esta investigación. (Rivero, 2013).

Desde hace pocos años se consideraba que el uso de las computadoras estaba exclusivamente para alumnos mayores, pero ya existen diferentes iniciativas en la que la incorporación de las TIC en la Educación Inicial. Muchos educadores ven el uso de los computadores en estos primeros cursos, como un medio de involucrar a los niños con las labores académicas. Es por ello, que el uso temprano las TIC nos permite que los alumnos jóvenes se familiaricen con la Tecnología, lo cual coincide con el planteamiento del componente tecnología y calidad de vida del Currículo de Educación Inicial, que se refiere a la relación entre la tecnología y el desarrollo de habilidades en los niñas y niños como apoyo al aprendizaje y como base para el uso en la vida diaria. León, \& Pacheco, (2014).

Poco a poco las TIC se han ido incorporado en el ámbito escolar, como medio para facilitar el proceso de enseñanza y aprendizaje, de los alumnos como una herramienta de apoyo en los docentes, los cuales han incluido estas innovaciones las aulas, actualmente el uso de las nuevas tecnologías han captado la atención de los alumnos por completo, lo cual las mismas han sido llevadas en el ámbito de la educación, por lo que nos presenta las distintas maneras de utilizarlas y llevarlas a cabo en el proceso de enseñanza y aprendizaje de los alumnos. Es por ello, que la tecnología ha llegado a las instituciones educativas, donde los alumnos por medio de los docentes van conociendo cuales son los diferentes tipos de tecnología con los cuales se pueden ayudar para llevar a cabo un mejor aprendizaje. Por consiguiente, la integración de las TIC en la educación en el bachillerato, ha sido una innovación importante para la sociedad puesto que los estudiantes aprenden de una manera mucho más significativa, didáctica y moderna. León, \& Pacheco, (2014). 
Aunque durante el desarrollo de nuevas metodologías y métodos de enseñanza se ha englobado a las TICs como una herramienta eficaz y eficiente para el desarrollo de la enseñanza aprendizaje, incluidas en estas las redes sociales, no podemos asegurar que su implementación sea solución para los problemas educativos, por cuanto existen muchos factores de carácter externo que pueden provocar una distracción de la finalidad especifica de educar. (Sanz, 2008).

En la actualidad, se destina mucho tiempo a la interacción en redes sociales; según un estudio argentino de 2010, señala que la tasa de uso de internet entre adolescentes de -12 a 19 años en redes sociales específicamente- es muy alta, y aunque no es nuestra realidad, podemos inferir similitudes en el comportamiento social adolescente latinoamericano. (Morduchowicz, 2010).

Se logra establecer la influencia e incidencia de las redes sociales en la educación de adolecentes de una entidad educativa ecuatoriana. Considerando que esta generación y las redes sociales se han convertido en una plataforma de comunicación global, es muy fácil que se tornen hasta cierto punto adictivas, y por esto se han convertido en distractores para los estudiantes. A través de datos estadísticos de carácter histórico, se compararon los promedios de aprovechamiento de cada uno los sujetos de estudio durante su permanencia en la secundaria, en Matemáticas y Física, desde su educación básica superior (octavo, noveno y décimo año) hasta el bachillerato general unificado, contrastado con el tiempo dedicado a navegar en el internet visitando paginas como Facebook, Instagram, Messenger, Google +, Skype, Hi5, Twitter, WhatsApp, YouTube, etc. Esto permitió determinar la influencia que las redes sociales tienen en las actividades diarias de los estudiantes, quienes dedicaron la mayor parte de su tiempo de estudio autónomo a chatear, incidiendo de forma negativa en el rendimiento académico de los sujetos de estudio. Bustos, et al., (2016). 


\section{Conclusiones.}

La función más importante de las instituciones de educación en general, con respecto al papel de la pedagogía dentro de las mismas es ir configurando un proceso bastante extenso en el que los conocimientos se integren hasta estructurar los saberes de cada persona. Es importante que los educandos retomen las ideas que los tutores y tutoras imparten frente a los motivos que ocasionan determinadas situaciones, pero estas no deben desarrollarse solamente desde un referente teórico, por lo que es trascendental realizar las prácticas de manera cotidiana con el propósito de confrontar lo que se dice con lo que se hace, de este modo buscar las inconsistencias, las rupturas, las tenciones y las coincidencias y desde esta actividad estructurar un marco explicativo de la vida.

El éxito académico en las instituciones educativas, dependen de los hábitos y técnicas de estudio que se emplean en la institución, así como también, en casa. En tal sentido, es muy significativo contar con un adecuado ambiente y materiales necesarios para que el estudio sea una actividad exitosa, reconociendo, que el docente mediante su desempeño es el eje que moviliza el proceso de formación dentro del sistema educativo formal, siendo muy significativa su formación inicial y la capacitación continua y permanente.

Por tal motivo, se tiene que las competencias que generan atributos para el desempeño del docente en situaciones diversas dentro del campo educativo son de suma importancia. Adicional a esto, y durante el desarrollo del trabajo de investigación se hace mención, al uso de material didáctico, en el trabajo educativo, lo que permite desarrollar caracteres importantes que intervienen y facilitan el proceso de enseñanza-aprendizaje.

Los materiales didácticos tanto físicos como virtuales, asumen, despertar el interés de los estudiantes, adecuándose a las características físicas y psíquicas de los mismos, además que facilitan 
la actividad docente al servir de guía, gracias a la virtud de adecuarse a cualquier tipo de contenido. El acceso a los diferentes recursos TIC, ofrecen un entorno mucho más favorable para el aprendizaje y una experiencia docente más dinámica, de la misma manera, las TIC para los estudiantes son fuertemente motivadoras y brindan aprendizajes más activos. Su uso en el aprendizaje basado en proyectos y en trabajos grupales permite tener acceso a recursos, que conlleva a una enseñanza más creativa tanta para los docentes como para los estudiantes. La incidencia de las redes sociales en la educación de adolecentes se ha transformado en una plataforma de comunicación global, sin embargo, su uso debe ser supervisado porque puede convertirse en distractores para los estudiantes, haciendo que no se cumpla con la finalidad específica de educar.

\section{Bibliografia.}

Ávila, P. (2011). Ambientes virtuales de aprendizaje una nueva experiencia. Alemania: Dusseldorf.

Belaunde, I. (1994). Hábitos de estudio. Revista de la facultad de psicología de la universidad femenina de sagrado corazón.

Brunet, F. (2007). Factores que afectan los hábitos de estudio en los escolares de primaria. Buenos Aires: Bruño.

Brunet, G. (2005). Técnicas de lectura eficaz, Editorial Bruño. Madrid.

Bustos G., A. F. (2016). Las redes sociales, su influencia e incidencia en el rendimiento académico de los estudiantes de una entidad educativa ecuatoriana en las asignaturas de Física y Matemática. Lat. Am. J. Phys. Educ. Vol. 10, No. 1, ISSN 1870-9095. Obtenido de http://www.lajpe.org

Cárdenas, H. (2008). Compilación y Adaptación. Quito.

Chiavenato, A. (1984). Introducción a la teoría general de la administración, Tercera edición (segunda en español). Bogota: Mc. Graw Hill.

CONESUP, M. d. (2006). La Formación Inicial de Educación Básica en los Institutos Superiores Pedagógicos. Quito, Ecuador.

Crespo, N. (2010). La clave del éxito en todo el proceso educativo, Ministerio de Educación del Ecuador. Ecuador. 
Echeita, G. y. (2011). La educación inclusiva como derecho. Marco de referencia y pautas de acción para el desarrollo de una revolución pendiente en Red de información educativa núm. 12, Tomado http://redined.mecd.gob.es/xmlui/bitstream/handle/11162/29104/00920113014346.pdf?seq. Tejuelo.

Estrada, L. (2013). El desempeño docente, Universidad de Carabobo, Carabobo - Venezuela. Carabobo, Venezuela.

Fabara, E. (2013). Estado del arte de la formación docente en el Ecuador, Cuadernos del Contrato Social por la Educación $N^{\circ} 8$. Quito, Ecuador.

Flores, T. (2011). Fundación Fidal.

Giménez, C. (2003). Pluralismo, multiculturalismo e interculturalidad propuesta de clarificación y apuntes educativos, núm. 8, Editorial CES Don Bosco-EDEBË. http://es.scribd.com/doc/110048989/Pluralismo-Multiculturalismo-eInterculturalidad\#scribd. Revista Educación y Futuro. Revista de Investigación Aplicada y Experiencias Educativas, 9-26.

Gómez, M. (2011). El modelo educativo basado en competencias en el bachillerato mexicano y el desarrollo de habilidades de información. Revista Chilena de Bibliotecología, Infoconexión Número 2 .

Góngora, C. (2010). Metodo para estudiar . Obtenido de metodoparaestudiar.blogsport.com/2008/01/hbitos-de-estudio.htm

Guzmán, R. G. (2010). Concepciones de infancia, alfabetización inicial y aprendizaje de los $\begin{array}{llllll}\text { educadores } & y & \text { educadoras, } & \text { vol. } & 8, & \text { núm. }\end{array}$ http://www.umanizales.edu.co/revistacinde/index.html. Revista Latinoamericana de Ciencias Sociales Niñez y Juventud, 861-872.

Hernández, C. (1999). proximaciones a la discusión sobre el perfil del docente. II Seminario Taller sobre perfil docente y estrategias de formación. San Salvador.

Herrera, M. (2010). Ambientes virtuales de aprendizaje. Obtenido de http://www.rieoei.org/deloslectores/1326Herrera.pdf

Montolio, L. y. (2008). Una escuela de todas (las personas) para todas (las personas), en, vol. 6, núm. 2, disponible en: http://www.rinace.net/arts/vol6num2/art8.pdf. Revista Electrónica Iberoamericana sobre Calidad, Eficacia y Cambio en Educación REICE.

Morduchowicz, R. (2010). Los adolescentes y las redes sociales, Fondo de Cultura Económica., (pág. 7). Mexico.

Nava, H. (2009). Instituciones educativas y cultura escolar, Villa de Álvarez, Colima. Obtenido de www.buenastareas.com/.. Comportamiento-Institucional/447744.html

Ogalde, O. (2008). Libro de materiales didácticos. Barcelona: Gedisa. 
Ossa, G. (2002). Tendencias Educativas para el siglo XXI, Educación virtual, online y @learning, Elementos para la discusión, . Obtenido de Disponible en: http://edutec.rediris.es/Revelec2/revelec15/cardona.pdf

Parrilla, A. (2002). Acerca del origen y sentido de la educación inclusiva, Número 327. Tomado de http://www.mecd.gob.es/dctm/revista-deeducacion/articulos327/re3270210520.pdf?documentId=0901e72b81259a76. Revista de Educación, 11-29.

Pérez, Á. (1998). La cultura institucional de la escuela. Cuadernos de Pedagogía, 80-82.

Pilco, N. (2013). La utilización de los recursos didácticos en la enseñanza aprendizaje de la matemática y su incidencia en el rendimiento académico de los estudiantes del segundo año de bachillerato general unificado del colegio "Amelia Gallegos Díaz". Titulo de pregrado, Universidad Nacional de Chimborazo, Riobamba, Ecuador. Ecuador.

Rivadeneira, E. (2012). Perfil de competencias de la formación docente en los institutos superiores pedagógicos del país. . tesis de maestría, Universidad Andina Simón Bolívar. Ecuador.

Rivera, M. (2011). Materiales didácticos del nivel inicial. Obtenido de Disponible en: http://masiellriverac.blogspot.com/2011/11/materiales-didacticos-del-nivel-inicial.html

Rivero, I. (2013). Criterios para seleccionar tecnologías educativas y estrategias didácticas en el Colegio Guillermo León Valencia. En Educación y ciencia. (págs. 37-52).

Robalino, M. (2007). Los docentes pueden hacer la diferencia: apuntes acerca del desarrollo profesional y el protagonismo docente, Santiago, Chile. Santiago de Chile.

Rodríguez, N. (2004). El clima escolar, Revista $\mathrm{N}^{\mathrm{o}}$ 7, volumen 3, disponible en: www.c.sif.es/andalucía/modules/mod_sevilla/archivos/revistaense/n7v3/clima.pdf. Revista digital "Investigación y educación.

Romero, M. (2002). La Reforma Educativa: del papel a la realidad ¿Qué está ocurriendo en los espacios de coordinación del Plan 96? . Tesis de Maestría UCU. Montevideo.

Sanz, A. G. (2008). Las redes sociales como herramientas para el aprendizaje colaborativo: Una experiencia con Facebook, Representaciones. Estudios de Periodismo, Comunicación y Sociedad 5, 49-59.

SEP, S. d. (2006). Los Materiales Educativos en México: Aproximación a su génesis y desarrollo, Miembros del Seminario Los materiales educativos, en la sociedad de la información. Mexico.

Torres, J. (2008). Gestiopolis. Obtenido de http://www.gestiopolis.com/organizaciontalento/gestion-del-desempeno-y-su-comportamiento.htm

UNESCO. (2002). Educación para Todos: Cumplir nuestros compromisos comunes. Tomado de: http://unesdoc.unesco.org/images/0012/001211/121147s.pdf. Foro Mundial sobre la Educación. Dakar, Senegal. 
Lady D. Vera-Solórzano; Luz M. Samaniego-Bautista; Edison C. Maldonado-Alvarado; Andrea C. Pabón-Soria; Andrea C. Loachamin-Guachamin; Katty A. Chariguaman-Chanaluisa

Valdés, H. (2004). El desempeño del maestro docente y su evaluación, Pueblo y Educación. La Habana.

Vargas, M. (2003). Materiales educativos, procesos y resultados. Obtenido de Disponible en: http://books.google.com.ec/books?id=KG3I5RaTwO4C\&pg=PA180\&lpg=PA180\&dq=mate riales $+\operatorname{did} \% \mathrm{C} 3 \%$

Villalobos, L. (2010). Blog Observatorio parámetro. Obtenido de Recuperado de: http://contenidoparametro-obs.blogspot.com/2010/01/gestion-pedagogica-en-relacion-al.html

Viñao, A. (2002). Sistemas educativos, culturas escolares y reformas, Morata. Madrid. 